\title{
Redes de consejo en la industria biotecnológica en Francia $^{1}$
}

\author{
Alvaro Piña-Stranger ${ }^{2}$ - IRISSO (CNRS - Université Paris Dauphine) ${ }^{3}$ \\ Emmanuel Lazega - IRISSO (CNRS - Université Paris Dauphine)
}

\begin{abstract}
Resumen
En este artículo exploramos las redes de consejo inter-organizacionales, apoyándonos en la idea de que la naturaleza de las interdependencias funcionales entre los actores es diferente a nivel intra-organizacional y a nivel interorganizacional. Esta diferencia influye en la manera en la que los miembros de un colectivo tratan de resolver los problemas relacionados con la evaluación de la pertinencia de los conocimientos. A nivel intra-organizacional, los conflictos epistémicos se resuelven por un movimiento de centralización y de alineamiento en torno a algunos pocos actores que detentan una autoridad formal. Esta hegemonía, formal y cognitiva, les permite imponer un consenso y ejercer un control elevado sobre el colectivo. En cambio, a nivel inter-organizacional, los conflictos epistémicos se resuelven por un movimiento de polarización, que es mantenido en parte por el activismo relacional de varios líderes de opinión, cuyo poder coercitivo no es absoluto. Ilustramos este proceso de aprendizaje a través de las redes de consejo entre empresarios científicos en la industria biotecnológica francesa.
\end{abstract}

Palabras clave: aprendizaje colectivo - homofilia - polarización social - líderes de opinión.

\begin{abstract}
In this article we explore inter-organizational advice networks to build on the idea that the nature of the functional interdependencies between actors is different at the intra-organizational and the inter-organizational level. This difference influences the way in which members of a collective attempt to solve problems associated with evaluating the relevance of knowledge. At the intra-organizational level, cognitive conflicts are resolved by a movement of centralization and alignment around a few actors who benefit from formal authority. This hegemony, formal and cognitive, allows them to impose a consensus and to exercise high control over the collective. In contrast, this paper shows that at the inter-organizational level, cognitive conflicts are resolved by a movement of polarization, which is maintained in part by the relational activism of several opinion leaders whose coercive power is not absolute. We illustrate this learning process through advice networks between scientific entrepreneurs in the French biotechnology industry
\end{abstract}

Key words: collective learning - homophily - social polarization - opinion leaders

\footnotetext{
1 Agradecemos al European Journal International Management (www.elsevier.com/locate/ejim) por permitirnos presentar en este artículo algunos de los resultados presentados en Pina-Stranger, A. and Lazega, E. (2010) 'Inter-organisational collective learning: the case of biotechnology in France', European J. International Management, Vol. 4, No. 6, pp.602-620.

2 Enviar correspondencia a: Álvaro Piña-Stranger alvaro.pina-stranger@dauphine.fr

${ }^{3}$ Los autores agradecen el apoyo de la Ville de Paris que hizo este estudio posible, y a Omar Sabaj por sus comentarios y correcciones.
} 


\section{I ntroduction}

El aprendizaje colectivo es de suma importancia para el funcionamiento y el desempeño económico de las empresas. El rápido desarrollo de la industria del conocimiento sitúa a los saberes y sus dinámicas de creación y de difusión en el centro de las preocupaciones de las autoridades públicas encargadas de facilitar la innovación industrial. El desarrollo de los métodos de análisis de redes sociales ha dado un nuevo impulso a los trabajos en sociología económica que se interesan en estos procesos, permitiéndoles captar de mejor modo la complejidad relacional e interactiva de las dinámicas que se ponen en juego en este ámbito.

Los primeros estudios sobre el aprendizaje colectivo se centraron en la descripción de las relaciones e interacciones al interior de las organizaciones. Las propuestas de Blau (1955, 1964), para quien los actores se ven incitados a compartir sus conocimientos si obtienen a cambio el reconocimiento de su autoridad epistémica, sirvieron de marco teórico a estos trabajos. Al examinar las relaciones de consejo, estos trabajos explicitaron los "juegos de status" y los mecanismos de alineamiento epistémico a través de los cuales un colectivo hace posible el aprendizaje de sus miembros. Se pudo establecer así, que en las organizaciones la aparición de una estructura jerárquica, a menudo conectada a la estructura formal de la organización, otorga al colectivo la capacidad de evaluar y controlar los conocimientos que pueden considerarse pertinentes para la realización de las actividades.

Recientemente, un gran número de estudios se han interesado en la construcción y la transferencia de conocimientos a nivel inter-organizacional, en particular, en los nuevos sectores de innovación industrial. Adoptando una concepción relacional del conocimiento, estos trabajos examinan el contexto inter-organizacional en el cual se integran los proyectos innovadores con el fin de estudiar los mecanismos comerciales y públicos de valorización científica. En la industria biotecnológica, distintos autores (Powell et al., 1996, Senker et Sharp, 1997, Baum et al., 2000, Salman et Saives, 2005) han explorado el efecto de las colaboraciones interorganizacionales en la capacidad de las empresas para desarrollarse, innovar o aprender. Estos trabajos se interesan por las formas de intercambio que mantienen las empresas. En la síntesis teórica propuesta por Kogut (2000), se sugiere que las relaciones inter-organizacionales tienden hacia la estabilización de una norma de intercambio dominante. La ausencia de una autoridad formal es compensada por la aparición de comunidades que se auto organizan, haciendo posible la cooperación 
entre los distintos actores. Sin embargo, es importante no perder de vista que lo que está en juego en la competición que se instaura entre las distintas normas no se reduce a las modalidades de intercambio que estas reivindican. Esta competición dice relación también con los objetivos, los valores y las identidades que definen el papel de un colectivo en un sistema inter-organizacional más amplio y políticamente determinado. La dimensión simbólica de los intercambios representada por estos elementos determina también la estructuración de un sector de producción.

Tomar en cuenta seriamente esta dimensión no económica es uno de los principales retos del enfoque neo-estructural (Lazega et Mounier, 20). Este enfoque supone observar las relaciones sociales que mantienen los actores a nivel interindividual. En la industria biotecnológica, distintos trabajos en economía-geográfica ponen en evidencia que distintos entornos físicos e institucionales (universidades, clústers tecnológicos, asociaciones profesionales, etc.) permiten la interacción entre empresarios científicos (Audretsch y Stephan, 1996, Lemarié y am., 2001, Powell y al., 2002, Stuart y Sorenson, 2003, Autant-Bernard y al., 2006). Las relaciones interindividuales dan cuenta de un sistema de intercambio informal complejo donde se establecen distintos tipos de interdependencias. El estudio de estas interdependencias permite situar a los individuos en el centro de las explicaciones sobre el funcionamiento social del aprendizaje inter-organizacional.

En este artículo exploramos el aprendizaje inter-organizacional a través de las relaciones de consejo que mantienen los empresarios científicos en biotecnología. Nos basamos en la idea de que la naturaleza de las interdependencias funcionales entre los actores es diferente a nivel intra-organizacional y a nivel interorganizacional. Esta diferencia tiene implicaciones sobre la manera en la que el colectivo soluciona los conflictos epistémicos vinculados a la jerarquización de las autoridades y los conocimientos pertinentes. En la próxima sección, proponemos en primer lugar un marco teórico para distinguir la naturaleza de las interdependencias en estos diferentes niveles de análisis, así como las consecuencias de estas diferencias sobre el proceso colectivo de aprendizaje. A continuación, derivamos de esta teoría las hipótesis relativas a la estructura y el funcionamiento de este proceso a nivel inter-organizacional. Por último, apoyándonos en un estudio empírico realizado sobre los empresarios de la industria biotecnológica en Francia, probamos nuestras hipótesis y discutimos nuestros resultados así como los límites de nuestro enfoque. 


\section{Ausencia de autoridad formal y transferencia de conocimientos a nivel inter-organizacional}

El análisis de las relaciones de consejo a nivel inter-organizacional que dan cuenta de la transferencia de conocimientos, puede ser útil para el estudio de la relación entre la estructura social de un sector de producción y los comportamientos de los empresarios.

Si numerosos trabajos se han interesado por las relaciones de consejo en contextos profesionales, es porque la reconstitución de este tipo de interacciones sociales permite articular las dos dimensiones de los intercambios: relacional y simbólica. Por una parte, ser elegido como consejero es un indicador de los recursos que un actor controla. Por otra parte, elegir a un consejero es concederle un determinado status epistémico. La primera dimensión hace referencia a los recursos que circulan en un colectivo, mientras que el segundo hace referencia a los compromisos personales, a las identidades y valores que permiten a los actores politizar y contextualizar sus intercambios. Considerar estas dos dimensiones equivale a postular que los juicios a partir de los cuales los actores evalúan los conocimientos que deben tomarse en cuenta para la realización de las tareas, se negocian en función de criterios técnicos de satisfacción, pero también en función de criterios sociales de pertinencia (Lazega, 1992). Estos juicios de pertinencia orientan los comportamientos en la medida en que permiten a los actores jerarquizar a los individuos y a los grupos a los que se les atribuye una cierta autoridad de saber, esto es, el derecho a saber. El actor se alinea epistémicamente con una autoridad $y$, como contrapartida, espera ser reconocido como uno de los miembros de la comunidad en la cual esta autoridad se ejerce. La agregación de estas distintas elecciones relacionales permite hacer emerger una estructura donde es posible identificar las comunidades epistémicas que conforman un medio social.

Los juegos de status que orientan la evaluación y la selección colectiva de los conocimientos se han observado en sistemas de interdependencias funcionales. Estas interdependencias configuran las oportunidades y las dificultades que pesan sobre las decisiones de los actores. A la escala inter-organizacional, estas interdependencias son la mayor parte del tiempo bien definidas, pues la división del trabajo está relativamente clara. Distintos estudios ponen de manifiesto a este nivel de análisis que las redes de consejo tienden a ser jerárquicas. Esta jerarquía informal coincide a menudo con la estructura formal de la organización: los actores que presentan un elevado status epistémico son aquellos que ejercen una autoridad 
formal (Lazega et al., 2006b). Por una parte, las interdependencias impuestas por la estructura organizacional incentivan a los actores a una forma de conformidad frente a los juicios reivindicados por las autoridades formales, garantizando así su soberanía epistémica. Por otra parte, el manejo de esta posición de autoridad incita a estos líderes de opinión a no solicitar el consejo de aquellos actores menos reconocidos que ellos.

A nivel inter-organizacional, los trabajos sobre el aprendizaje colectivo se concentran a menudo sobre las interdependencias contractuales de las empresas. En la industria biotecnológica, numerosos investigadores han examinado la posición de las empresas en la red de colaboraciones inter-organizacionales. Entre los principales resultados de estas investigaciones podemos indicar: la importancia del número y la diversidad de las colaboraciones para el crecimiento de las empresas (Powell y al., 1999); el impacto positivo de las colaboraciones con centros de investigación académica sobre sus capacidades de innovación (Baum y al., 2000, Autant-Bernard y al., 2006, Stuart y al., 2007); y el efecto motor de la proximidad geográfica de los colaboradores sobre la creación de empresas (Powell y al., 2002, Boufaden y al., 2005, Gertler, 2005, Aharonson y al., 2008, Gilding, 2008). Estos resultados han permitido describir la estructura económica de esta industria, y algunas de las normas de intercambio que orientan su desarrollo.

Kogut (2000) sugiere que a pesar de la ausencia de una autoridad formal, las relaciones inter-organizacionales tienden hacia la homogeneización de una norma que domina los intercambios. Esto supone que otras normas no obtuvieron el apoyo necesario para imponerse. El estudio de las relaciones que mantienen los individuos implicados en la construcción de estas normas, permite explorar bajo qué condiciones sociales una forma de intercambio se vuelve predominante. Como lo indican varios trabajos sobre el sector biotecnológico (Pisano, 1991, Casper, 2007, Niosi, 2003, Mangematin y al., 2003, Powell y al., 1996), el intercambio personalizado de ideas fuera de las fronteras organizacionales es determinante para las empresas. La envergadura de las actividades de investigación y desarrollo $(I+D)$, el cambio rápido de las dificultades vinculadas al reglamento sanitario, así como el carácter no codificado de los conocimientos movilizados por las empresas configuran un medio ambiente extremadamente incierto. Esto anima a los empresarios a solicitar e integrar al consejo de sus pares competidores en sus procesos de toma de decisiones. Ahora bien, la elección de los consejeros no es anodina, ella resulta de la evaluación que hacen los actores de lo que está en 
juego, a nivel simbólico y económico, en la negociación de las normas y conocimientos reivindicados por las distintas comunidades epistémicas.

Contrariamente a las relaciones intra-organizacionales, la elección de los consejeros a nivel inter-organizacional no está determinada directamente por las interdependencias funcionales de una estructura formal común. El marco de estas interacciones es diferente en la medida en que, a nivel inter-organizacional, el peso de las afiliaciones organizacionales sólo tiene un poder coercitivo indirecto sobre los actores. Es oportuno preguntarse entonces cómo, en un contexto en el que las formas de regulación formales no participan en la coordinación de los intercambios, el colectivo soluciona los conflictos epistémicos vinculados con la evaluación de los conocimientos pertinentes y la definición de las normas legítimas.

Los estudios sobre las relaciones de consejo a nivel intra-organizacional muestran que los conflictos epistémicos se solucionan por una fuerte alineación epistémica y un movimiento de centralización en torno a los miembros que se benefician de una autoridad formal. La alineación de la mayoría con la posición de un número limitado de líderes de opinión permite a estos últimos orientar el proceso de aprendizaje imponiendo un consenso y efectuando un control epistémico sobre el colectivo (Lazega y al. 2006b). En cambio, a nivel inter-organizacional, el déficit de interdependencias funcionales deroga hasta cierto punto el valor de las autoridades formales en el juego de status entre los actores. En este sentido, estamos ante un sistema "más colegial" que "burocrático" puesto que, en principio, todos los actores pueden participar de la definición de las reglas del juego (Raynaud, 1989, Lazega, 1999), y, en particular, de las negociaciones destinadas a definir los términos que rigen los intercambios. Durante estas negociaciones, los actores se reconocen mutuamente como miembros de una misma comunidad en función de criterios de identidad como la homofilia, los status epistémicos o los intereses comunes. Así pues, a nivel inter-organizacional, la precariedad de las autoridades formales favorece la aparición de distintas comunidades epistémicas que luchan por mejorar la posición de sus miembros, promoviendo algunos conocimientos o normas de intercambio por sobre otros.

Las relaciones sociales que mantienen los productores en un sector de actividad se caracterizan por esta dimensión colegial de los intercambios. En este sentido, los conflictos asociados al aprendizaje colectivo no se solucionan de la misma manera que a nivel intra-organizacional. En vez de una estructura jerárquica en donde un único subgrupo de actores domina e impone los juicios que reivindica, la naturaleza más política que funcional de las interdependencias a nivel inter-organizacional se 
traduce en una polarización del colectivo en torno a varios líderes de opinión cuyo poder coercitivo no es absoluto. Su legitimidad, así como la de los conocimientos y normas de intercambio que reivindican, son objeto de un conflicto ininterrumpido. El déficit reglamentario formal que caracteriza las relaciones inter-organizacionales causa así un debilitamiento del status epistémico de los empresarios que han obtenido o que quieren obtener la autoridad de saber. En este contexto, el control que el medio es capaz de imponer a los líderes de opinión exige de su parte una mayor inversión política destinada a mantener y renovar las relaciones que sostienen su posición privilegiada. Estas inversiones relacionales se crean como una estrategia de compensación ante el déficit de autoridades formales que permiten a las élites, a nivel intra-organizacional, de garantizar su supremacía.

\section{Hipótesis}

Esta teoría nos permite formular tres hipótesis relativas a la estructura del sistema de intercambio de consejo a nivel inter-organizacional y a algunas de las características que orientan su funcionamiento.

Acabamos de ver que el déficit de autoridades formales en las relaciones interorganizacionales favorece un movimiento de polarización en el colectivo. Derivamos de esta propuesta nuestra primera hipótesis. El análisis conjunto de las relaciones de consejo y de los atributos de los actores debería hacer surgir diferentes subgrupos cohesivos que mantienen pocas relaciones los unos con los otros. Los criterios de identidad (exógenos y endógenos) representados por estos atributos deberían guiar la elección de los consejeros: los actores que comparten los mismos atributos tenderán a elegirse más entre ellos que con los miembros de los otros subgrupos.

Nuestra segunda hipótesis tiene por objeto explorar las subestructuras que caracterizan la red de consejo. Nuestra primera hipótesis supone la constitución de distintos polos o comunidades, al interior de los cuales, las elecciones relacionales se centralizan en torno a diferentes líderes de opinión. En términos estructurales, esto significa que la red debería caracterizarse a la vez por la presencia de subestructuras transitivas y por subestructuras que den cuenta de una distribución jerárquica de las elecciones. 
Por último, hemos visto que la existencia de varios polos debilita el status epistémico de los actores que han adquirido la autoridad de saber. A nivel intraorganizacional, donde estos status están "protegidos" por la estructura formal de la organización, se observa generalmente una correlación negativa entre las elecciones recibidas y las elecciones emitidas. A nivel inter-organizacional esta protección formal es deficitaria. Predecimos que el comportamiento de los actores destinados a compensar este déficit se traduce en una correlación positiva entre las elecciones recibidas y emitidas: los actores más elegidos deberían ser los que solicitan más consejos, esto es, los mas populares son también los más activos.

\section{Método de análisis}

Con el fin de estudiar el efecto de los atributos exógenos y endógenos sobre la demanda y la atribución de consejos, hemos movilizado los modelos estadísticos p2 y ERGM (Siena). El modelo p2 (Lazega y Van Duijn, 1997, Van Duijn y al., 2004, Zijlstra y Van Duijn, 2008) permite analizar redes binarias orientadas. Su principal ventaja es que considera las elecciones emitidas y recibidas por un mismo actor como interdependientes. El modelo permite incluir los atributos de los actores como variables explicativas. Utilizamos este modelo para probar el efecto de las desemejanzas de atributo sobre la ocurrencia de las relaciones (efecto de densidad) entre los actores. Para ello, hemos transformado los atributos categoriales de los actores en variables diádicas. Estas variables son matrices binarias en las que 1 indica que los actores pertenecen respectivamente a las dos categorías, y 0 indica que este no es el caso. Así pues, para cada atributo construimos distintas variables diádicas que nos permiten examinar el intercambio de recursos entre actores que pertenecen a categorías diferentes.

El modelo ERGM o p* (Snijders y al., 2006, Robins y al., 2007, Snijders y al., 2007) es un modelo estocástico concebido para identificar las subestructuras que caracterizan una red, incluyendo efectos aleatorios que representan la variabilidad de comportamientos que escapa a las observaciones. Las distintas características estructurales y exógenas (los atributos) están representadas por parámetros cuya estimación permite "configurar" el modelo. Se estima cada parámetro comparando sucesivamente la red observada a millares de redes simuladas aleatoriamente. La convergencia de estos parámetros indica la capacidad del modelo para ajustarse a la red observada. Por último, los parámetros estimados cuyo valor es significativo 
dan cuenta de las estructuras relacionales (y de las estructuras asociadas a atributos) que caracterizan la red y las elecciones relacionales de los actores. 


\section{Datos}

Los datos sobre los cuales exploramos nuestras hipótesis se recolectaron en el marco de una investigación realizada en 2008 sobre una subpoblación de dirigentes en biotecnología en Francia. Inicialmente, realizamos una investigación etnográfica donde entrevistamos a distintos tipos de actores (dirigentes, inversionistas, científicos y asesores) y realizamos numerosas jornadas de observación. Esto permitió definir y delimitar un conjunto de 96 empresas biotecnológicas dedicadas a la I+D en el sector de la salud humana. Estas empresas comparten al menos tres características:

a) las empresas trabajan en el ámbito de las ciencias de la vida;

b) las empresas han recibido inversiones de un importe superior a 500M€;

c) se trata de empresas francesas.

En función de su fase de desarrollo, incluimos entre 1 y 4 dirigentes por empresa, según los siguientes criterios:
a) fundadores;
b) presidente/director general;
c) director científico, financiero y de operaciones.

A continuación, creamos y probamos un cuestionario destinado a recoger información sobre sus trayectorias y sus relaciones con los otros empresarios para luego contactarlos y llevar a cabo las entrevistas. Conseguimos entrevistar a 138 empresarios. Hemos dejado un total de 164 dirigentes en nuestra población final, quienes representan 88 empresas biotecnológicas. La información relativa a los 26 actores que no desearon responder el cuestionario se obtuvo de bases de datos públicas.

\section{Variable dependiente}

La red de intercambio que estudiamos da cuenta de las relaciones de consejo que mantienen estos empresarios. Definimos tres tipos principales de actividades que corresponden al trabajo de colaboración que las empresas mantienen con sus principales socios: los centros de investigación académica, los inversores y las grandes empresas farmacéuticas. Los empresarios que estudiamos deben gestionar las relaciones con estos tres socios a lo largo de toda la vida de la empresa. Los 
centros de investigación son, a menudo, el lugar de origen de las empresas y de los científicos fundadores. Por su parte, los inversores proporcionan el dinero que permite a los empresarios desarrollar el proyecto científico en una estructura privada. Por último, las empresas farmacéuticas constituyen los principales clientes de las empresas biotecnológicas. Compran los productos en desarrollo o firman contratos de colaboración con ellas. Estos tres grandes sectores de actividad no son solamente los principales socios de las empresas biotecnológicas, ellos son en parte los principales actores que motivan su existencia (Arora et Gambardella, 1990, Pisano, 1991). Estos tres tipos de colaboradores coordinan sus intereses y recursos para crear las organizaciones que les permitirán aprovechar las oportunidades comerciales ofrecidas por este nuevo campo del conocimiento. En nuestro cuestionario, hemos pedido a los empresarios que nos indiquen a quienes solicitaban consejo sobre cuestiones relativas a la gestión de estos socios. De esta forma, hemos podido reconstituir, para cada tipo de socio, una red de consejo que representa el intercambio de recursos en el que son movilizados conocimientos asociados a ese socio en particular. La red de consejo que analizamos es una síntesis de las respuestas otorgadas para estos tres tipos de recursos.

\section{Variables independientes}

Incluimos en nuestros análisis seis atributos que representan distintos criterios de identidad (Tabla 1).

\begin{tabular}{|c|c|c|c|c|}
\hline Atributos de los actores & Tipo de variable & Ciencia & $\rightarrow \rightarrow \rightarrow \rightarrow \rightarrow \rightarrow \rightarrow \rightarrow \rightarrow \rightarrow \rightarrow \rightarrow$ & Economía \\
\hline Formación inicial & \multirow{2}{*}{ Binaria } & Científica & & Económica \\
\hline Número de actores & & 133 & & 31 \\
\hline Origen profesional & \multirow{2}{*}{ Categorial } & Investigación académica & Industria de la Salud & Finanzas \\
\hline Número de actores & & 50 & 84 & 30 \\
\hline Función en la empresa & \multirow{2}{*}{ Categorial } & Director científico & Presidente & Director Operaciones Director financiero \\
\hline Número de actores & & 23 & 88 & 20 \\
\hline \multirow{2}{*}{$\begin{array}{r}\text { Tipo de status epistémico } \\
\text { Número de actores }\end{array}$} & \multirow{2}{*}{ Categorial } & Científico & Generalistas & Económico \\
\hline & & 44 & 43 & 61 \\
\hline Rankin Indegree & Continua & \multicolumn{3}{|c|}{$\begin{array}{l}\text { Clasificación de los actores en una escala de } 1 \text { a } 10 \text { en función del numero de elecciones } \\
\text { recibidas }\end{array}$} \\
\hline Región & Categorial & \multicolumn{2}{|c|}{13 regiones están representadas } & \\
\hline
\end{tabular}

Tabla 1. Distribución de las variables independientes

Los dos primeros se refieren a las trayectorias de los empresarios. En la primera variable clasificamos a los empresarios en función de su "formación inicial". Distinguimos a los empresarios que seguían una "formación inicial científica", de los que siguieron una "formación inicial económica". La segunda variable representa el "origen profesional" de los actores. En ella hemos clasificado a los dirigentes en función del sector de actividad en el que trabajaban antes de integrar la empresa biotecnológica. Distinguimos a los actores procedentes de la industria 
biofarmacéutica, de los integrantes de centros de investigación y de los sujetos provenientes del sector financiero.

Los tres atributos siguientes se refieren a los status actuales (formales e informales) de los empresarios. En la tercera variable clasificamos a los empresarios según la función que ocupan en la empresa (status formal). La mayoría de los actores ocupa la función de "Presidente/Director General". Esta función es mayoritaria ya que está presente en todas las empresas, mientras que los "directores financieros", los "directores científicos" y los "directores de operaciones" integran la empresa una vez que esta ya registra un cierto crecimiento. La cuarta variable se construyó a partir del tipo de recurso sobre el cual los actores han sido solicitados (status informal). Clasificamos a los actores en tres grandes tipos de status epistémicos: el status "científico", en el que hemos incluido a los empresarios que han sido solicitados preferentemente para dar consejos sobre la gestión de las relaciones con los centros de investigación; el status "generalista", que agrupa a los empresarios que han sido elegidos para dar consejos sobre la gestión de los tres tipos de colaboradores de manera homogénea; y el status "económico", en el que hemos clasificado a los empresarios que han sido preferentemente elegidos para entregar consejos acerca de la gestión de los inversores. La quinta variable corresponde a la clasificación de los actores en función del número de elecciones recibidas en las 3 redes de consejo. Esta clasificación está representada por una escala de 1 a 10, dónde se ha asignado el valor 1 a los actores menos elegidos, y un valor 10 a los actores más elegidos. Por último, nuestra sexta variable representa las 13 regiones en las que se sitúan las empresas. Esto nos permitió controlar en nuestro modelo ERGM el efecto de la proximidad geografía en la ocurrencia de las relaciones de intercambio de conocimientos.

\section{Resultados}

Utilizamos estos datos para probar nuestras hipótesis sobre la estructura y el modo de funcionamiento del proceso de aprendizaje colectivo a nivel inter-organizacional. 


\section{Un proceso complejo de polarización social}

Nuestra primera hipótesis predecía que los actores que comparten los mismos atributos tenderían a establecer más relaciones entre ellos que con los demás actores. Para examinar esta hipótesis hemos realizado dos tipos de análisis. El primero atañe las relaciones que mantienen los actores que no tienen los mismos atributos, y el segundo, los actores que comparten el mismo atributo.

En primer lugar, analizamos el efecto de las disimilitudes de atributo relacionadas con "la función en la empresa", "el origen profesional" y el "tipo de status epistémico" de los actores, sobre el establecimiento de una relación de intercambio de consejo. Los Modelos p2 1 a 3, presentados en la Tabla 2, examinan el efecto de estas disimilitudes sobre la probabilidad de ocurrencia de las relaciones de consejo.

\begin{tabular}{|c|c|c|c|c|}
\hline & Modelo 1 & Modelo 2 & Modelo 3 & Modelo 4 \\
\hline Parámetros & $\begin{array}{c}\text { Diferencias de función en } \\
\text { la empresa }\end{array}$ & $\begin{array}{l}\text { Diferencias de origen } \\
\text { profesional }\end{array}$ & $\begin{array}{l}\text { Diferencias de status } \\
\text { cognitivos }\end{array}$ & $\begin{array}{l}\text { Diferencias transversales } \\
\text { "ciencia/economía" }\end{array}$ \\
\hline \multicolumn{5}{|l|}{ Efectos aleatorios } \\
\hline Variancia emisor & $1.22(0.21)$ & $1.21(0.20)$ & $1.23(0.21)$ & $1.23(0.21)$ \\
\hline Variancia receptor & $1.10(0.17)$ & $1.08(0.17)$ & $1.13(0.18)$ & $1.07(0.17)$ \\
\hline Covariancia emisor receptor & $0.01(0.13)$ & $-0.02(0.13)$ & $0.02(0.13)$ & $-0.01(0.13)$ \\
\hline \multicolumn{5}{|l|}{ Efectos globales } \\
\hline$\overline{\text { Densidad }}$ & $-3.91(0.14)$ & $-3.78(0.14)$ & $-2.62(0.26)$ & $-3.89(0.13)$ \\
\hline Reciprocidad & $2.23(0.18)$ & $2.40(0.19)$ & $1.30(0.91)$ & $2.58(0.15)$ \\
\hline \multicolumn{5}{|l|}{ Efecto de densidad específicos } \\
\hline Presidente - Dir. Financiero & $-0.58(0.15)$ & & & \\
\hline Presidente - Dir. Científico & $-0.22(0.19)$ & & & \\
\hline Presidente - Dir. Operaciones & $-0.06(0.11)$ & & & \\
\hline Dir. Financiero - Dir. Científico & $-1.59(0.46)$ & & & $-0,97(0,46)$ \\
\hline Dir. Financiero - Dir. Operaciones & $-0.81(0.28)$ & & & \\
\hline Dir. Científico - Dir. Operaciones & $-0.50(0.30)$ & & & \\
\hline Origen Finanza - Origen Academia & & $-0.82(0.17)$ & & $-0,35(0,17)$ \\
\hline Origen Finanza - Origen Salud & & $-0.51(0.13)^{*}$ & & \\
\hline Origen Academia - Origen Salud & & $-0.43(0.10)^{*}$ & & \\
\hline Status Económico - Status Científico & & & $-0.57(0.12)$ & $-0,48(0,12)$ \\
\hline Status Económico - Status Generalista & & & $-0.25(0.12)$ & \\
\hline Status Científico - Status Generalista & & & $0.05(0.14)$ & \\
\hline \multicolumn{5}{|l|}{ Efecto de reciprocidad } \\
\hline Presidente - Dir. Financiero & $1.08(0.32)$ & & & \\
\hline Dir. Financiero - Dir. Operaciones & $1.32(0.59)$ & & & \\
\hline Desviación & 5791.3760 & 5787.5162 & 5781.8698 & 5783.5092 \\
\hline
\end{tabular}

Tabla 2. Polarización de los intercambios en la red de consejo a nivel inter-organizacional

Tabla 2. Modelos p2. Efecto de las disimilitudes de atributo vinculadas a la "función en la empresa", el "origen profesional" y el "tipo de status epistémico" sobre las relaciones de consejo. Parámetros (desviación estándar). Los parámetros cuyo valor es al menos dos veces superior a la desviación estándar son considerados significativos. Las pruebas de ajuste (desviación) disminuyen del Modelo 1 al Modelo 3, indicando que los parámetros probados en el Modelo 3 se ajustan más a la red observada. 
En los tres primeros modelos observamos una preponderancia de valores negativos y significativos. Estos indican que cuando dos actores no comparten el mismo atributo, el establecimiento de una relación de consejo entre ellos es menos probable que la distribución aleatoria hecha a partir de sus características relacionales. Así pues, los criterios de identidad representados por estos atributos dividen al colectivo en varios subgrupos entre los cuales el intercambio de conocimientos es débil. Vale la pena indicar que entre los valores más significativos en los tres modelos encontramos a menudo la oposición "ciencia/economía": entre "directores financieros" y "directores científicos", entre "origen financiero" y "origen académico", y entre "status económico" y "status científico". El Modelo 4 examina en conjunto estas oposiciones. Los parámetros son todos negativos, indicando que los tres criterios de identidad representados por los atributos participan en el movimiento de polarización en esta red.

En segundo lugar, examinamos el efecto de las semejanzas de atributo sobre las relaciones de consejo. Para ello, se estimo un modelo $p^{*}$ presentado en la Tabla 3. Este modelo incluye los efectos estructurales vinculados a las subestructuras que caracterizan la red, los efectos exógenos vinculados a los atributos de los actores, y los efectos endógenos vinculados a su posición en el sistema de intercambio. 


\begin{tabular}{|c|c|c|c|c|}
\hline \multirow[b]{2}{*}{ Variables independientes } & \multicolumn{2}{|l|}{ Modelo 1} & \multicolumn{2}{|l|}{ Modelo 2} \\
\hline & $\begin{array}{c}\text { Parámetros y } \\
\text { desviación estándar }\end{array}$ & t-ratios & $\begin{array}{c}\text { Parámetros y } \\
\text { desviación estándar }\end{array}$ & t-ratios \\
\hline \multicolumn{5}{|l|}{ Efectos estructurales } \\
\hline Numero de lazos & \multicolumn{2}{|l|}{1231} & \multicolumn{2}{|l|}{1231} \\
\hline Densidad (parámetro fijo) & \multicolumn{2}{|l|}{0.04} & \multicolumn{2}{|l|}{0.04} \\
\hline Reciprocidad & $1.67(0.12)$ & 0.11 & $1.70(0.13)$ & 0.02 \\
\hline 3-cycles & $-0.08(0.03)$ & 0.07 & $-0.08(0.03)$ & -0.02 \\
\hline Alternating out-k-stars & $1.06(0.10)$ & 0.07 & $0.96(0.11)$ & 0.01 \\
\hline Alternating $\mathrm{k}$-triangles & $0.61(0.05)$ & 0.08 & $0.60(0.05)$ & -0.07 \\
\hline Alternating independent twopaths & $-0.06(0.01)$ & 0.10 & $-0.06(0.01)$ & 0.06 \\
\hline \multicolumn{5}{|l|}{ Atributos exógenos } \\
\hline Similitud de formación inicial & $0.08(0.04)$ & -0.04 & $0.11(0.04)$ & 0.04 \\
\hline Similitud de origen profesional & $0.16(0.04)$ & -0.10 & $0.17(0.05)$ & 0.05 \\
\hline Similitud de función en la empresa & $-0.02(0.04)$ & -0.07 & $-0.02(0.05)$ & -0.05 \\
\hline Similitud de región & $0.20(0.04)$ & -0.03 & $0.21(0.04)$ & 0.03 \\
\hline \multicolumn{5}{|l|}{ Atributos endógenos } \\
\hline Similitud de tipo de status epistémico & $0.15(0.05)$ & 0.09 & $0.17(0.05)$ & -0.04 \\
\hline Ranking Indegree ego & $0.04(0.02)$ & 0.11 & $0.06(0.02)$ & 0.08 \\
\hline Ranking Indegree alter & $0.19(0.02)$ & -0.01 & $0.20(0.02)$ & -0.11 \\
\hline Ranking Indegree similitud & $-0.40(0.14)$ & 0.10 & $-0.44(0.14)$ & 0.07 \\
\hline Actores que no respondieron ego & & & $-4.72(6.85)$ & 0.13 \\
\hline
\end{tabular}

Tabla 3. Alineación epistémica dentro de los polos, subestructuras e inversión relacional de las élites en la red de consejo a nivel inter-organizacional

Tabla 3. Modelo ERGM. Efectos estructurales, atributos endógenos y exógenos en la red de consejo a nivel inter-organizacional. Parámetros (desviación estándar). Los parámetros cuyo valor es al menos dos veces superior a la desviación estándar son considerados significativos. Las variables de los atributos relativos a las medidas de "similitud" son categoriales. La variable del atributo "ranking indegree" es una clasificación representada por una escala de 1 a 10, dónde se ha asignado el valor 1 a los actores que eran los menos elegidos, y un valor 10 a los actores que son lo más elegido como consejero.

Los parámetros de "similitud" miden la probabilidad de que los actores que comparten exactamente el mismo atributo establezcan una relación de consejo. Los valores de estos parámetros en el Modelo 1 ponen de manifiesto que tener el mismo "origen profesional" y el mismo "tipo de status epistémico" aumenta la probabilidad de establecer una relación de consejo. En el Modelo 2 hemos incluido una variable que representa a los actores que no desearon responder a nuestras preguntas. El efecto ego nos permite controlar las desviaciones producidas por la ausencia de datos relativos a las elecciones emitidas por estos 26 actores. El control de este efecto modifica ligeramente el modelo, pero permite obtener un 
valor del efecto de similitud positivo y significativo para los actores que siguen la misma formación inicial ("económico" o "científico"). El hecho de ocupar la misma función en la empresa no aumenta la probabilidad de establecer una relación de consejo. Esta variable es la menos significativa también en el Modelo 4 de la Tabla 2. Por último, el parámetro de la variable de control "similitud de región", que representa la tendencia a mantener relaciones con empresarios que trabajan en la misma región, es positivo y significativo. Pero la fuerte influencia geográfica sobre les elecciones relaciones que expresa este parámetro no alcanza a borrar el efecto de los otros atributos.

Estos resultados nos permiten confirmar nuestra primera hipótesis: las elecciones relacionales de los actores se ven orientadas por varias formas de homofilia que corresponden a los criterios de identidad representados por estos atributos. Vemos así que la dinámica de polarización del proceso de aprendizaje se base a la vez en la distancia relacional entre los distintos tipos de actores, y por una alineación epistémica dentro de los polos. Este fenómeno es complejo en la medida en que no se reduce a una única dimensión identitaria. El origen profesional, la formación inicial y los tipos de status epistémicos explican cada uno en parte el movimiento de polarización. Se trata de una característica de la estructura de interdependencias que se establecen en este medio pues en ella se articulan atributos exógenos y endógenos.

\section{Condiciones estructurales del movimiento de polarización}

Los primeros cinco parámetros del modelo ERGM (Tabla 3) corresponden a los efectos estructurales que representan las características de la red observada. Ellos nos permitirán explorar nuestra segunda hipótesis relativa a la presencia significativa de subestructuras transitivas. El valor del parámetro "reciprocidad" es positivo y significativo, lo que indica una fuerte tendencia de los actores a intercambiar consejos mutuamente. El parámetro "Alternating k-triangles" que es un indicador de transitividad representa la probabilidad de que los consejeros de mis consejeros se conviertan en mis consejeros. Este efecto asociado al parámetro negativo de la variable "alternating independent twopaths" indica que las relaciones de consejo tienden a organizarse en cliques, es decir, en distintos subgrupos más densos (Snijders y al., 2006). El efecto "alternating out-k-stars" es también positivo y significativo. Él representa la distribución de las elecciones emitidas, y confirma la fuerte probabilidad de ocurrencia de las subestructuras transitivas 
(Robins et Pattison, 2005). Pero, a diferencia de los "Alternating k-triangles", este indica una cierta segmentación en la red (Robins y al., 2007), confirmando así la tendencia indicada por el efecto 'alternating independent twopaths'. Por último, el valor negativo y significativo de la variable "3-cycles" (ciclos tríadicos) indica que es poco probable que los "aconsejados pidan consejo a los aconsejados sus de aconsejados". Esto sugiere la existencia de una tendencia relacional jerárquica subyacente a las tendencias transitivas más generales. Estos resultados confirman nuestra segunda hipótesis, las subestructuras transitivas, que son una condición relacional necesaria para la aparición de un movimiento de polarización, están representadas en la red de consejo. La red se caracteriza asimismo por una tendencia a la segmentación y por una "solidaridad limitada" que da cuenta de cómo los status epistémicos orientan también la elección de los consejeros.

\section{Comportamiento relacional de las elites a nivel inter-organizacional}

Nuestra última hipótesis predice una correlación positiva entre las elecciones emitidas y elecciones recibidas de los actores en la red de consejo. La Tabla 3 nos permite examinar visualmente esta correlación.

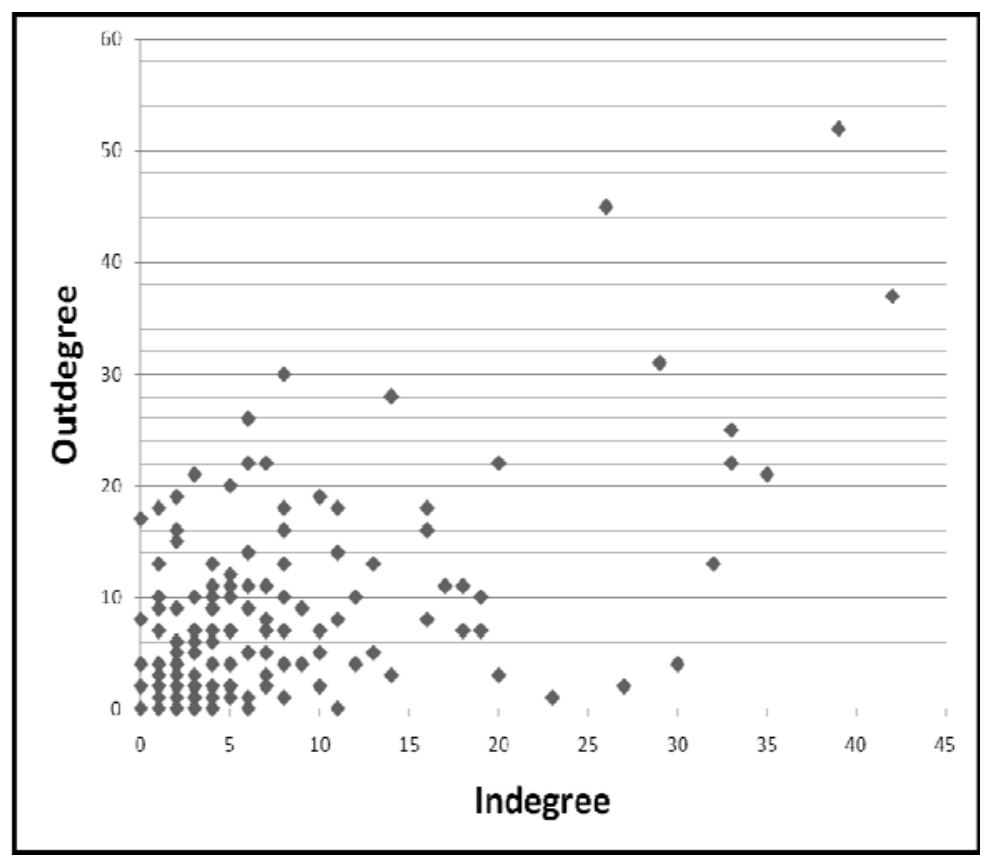

Tabla 4. Inversión relacional que caracteriza el comportamiento de las élites en la red de consejo a nivel inter-organizacional

Tabla 4. Correlación positiva entre las elecciones emitidas y elecciones recibidas en la red de consejo. Este gráfico representa el número de elecciones recibidas y emitidas de los 138 empresarios que respondieron al cuestionario sociométrico. La correlación entre las dos variables es de 0,545. 
Por una parte, observamos que las elecciones de los consejeros tienden a concentrarse en un puñado de actores $y$, por otra parte, que los actores que se ven beneficiados de un elevado status epistémico son también muy activos. Con el fin de comprobar estas observaciones incluimos en el modelo ERGM (Tabla 3) la variable "ranking indegree" dónde clasificamos a los actores (de 1 a 10) en función del número de elecciones recibidas. El efecto "ranking indegree ego" da cuenta de la relación entre el valor asignado a los actores en la clasificación y el número de elecciones que emitieron. El efecto es positivo y significativo indicando que los dos variable progresan en la misma dirección: cuanto más es elegido un actor, más este tenderá a elegir a otros actores. Esto confirma nuestra última hipótesis y pone de manifiesto que el comportamiento de las élites en esta red de consejo a nivel inter-organizacional se caracteriza por una fuerte inversión relacional. Por otra parte, el parámetro 'ranking indegree similarity', que es negativo y significativo, indica que los actores tienden a elegir como consejero de los actores que no tienen el mismo nivel de status que ellos, confirmando así la existencia de una estructura jerárquica. Por último, el parámetro 'ranking indegree alter' es naturalmente positivo y significativo.

\section{Discusión y conclusiones}

Exploramos en este artículo la estructura y el funcionamiento del aprendizaje colectivo a nivel inter-organizacional. Partimos de la idea de que la ausencia de un sistema formal compartido por los actores a nivel inter-organizacional tiene implicaciones sobre la manera en que un colectivo soluciona los problemas vinculados a la jerarquización de las autoridades y el conocimiento. A nivel intraorganizacional, el estudio de las redes de consejo ha puesto de manifiesto que, en un contexto donde las interdependencias funcionales están bien definidas, la elección de las autoridades epistémicas y de los conocimientos pertinentes está supeditada a la estructura jerárquica formal de la organización. En cambio, a nivel inter-organizacional, las afiliaciones organizacionales sólo tienen un poder coercitivo indirecto sobre los actores. En este contexto teórico, nuestras hipótesis nos han permitido explorar el efecto de una débil regulación formal sobre la estructura y el funcionamiento del proceso de aprendizaje inter-organizacional.

Nuestros resultados ponen de manifiesto que el carácter "colegial" de las relaciones inter-organizacionales favorece la aparición de distintas comunidades epistémicas alrededor de las cuales se alinean la mayoría de los individuos. Los actores orientan la elección de sus consejeros en función de distintos tipos de criterios de identidad. 
Las regularidades observadas en estas múltiples formas de homofilia dan cuenta de un movimiento de polarización, reforzado por un movimiento de alineación epistémico al interior de los polos. Como hemos mostrado en otros trabajos (PinaStranger, 2009, 2010), esta polarización parece basarse particularmente en la oposición "ciencia / economía". El análisis de las subestructuras que caracterizan la red de consejo confirma las siguientes tendencias: observamos a la vez una sobrerrepresentación de las subestructuras transitivas, y un orden jerárquico que caracteriza la elección de los consejeros. Por último, hemos mostrado que, contrariamente a lo que se ha observado al interior de las organizaciones, el comportamiento de las élites en esta red inter-organizacional se caracteriza por una fuerte inversión relacional. Nuestro estudio sugiere que este comportamiento corresponde a una estrategia de compensación destinada a reforzar la posición de los actores en un medio ambiente polarizado en el que los conflictos que emanan de las otras comunidades epistémicas debilitan el status cognoscitivo de los empresarios que conquistan la autoridad de saber. En resumen, hemos mostrado que la elección de las autoridades epistémicas y de los conocimientos pertinentes a nivel inter-organizacional se efectúa a través de un movimiento colectivo de polarización, mantenido, en parte, por el activismo relacional de las élites.

El fenómeno de polarización social que acabamos de estudiar impide la creación de un marco común que facilite los intercambios en el colectivo. En este sentido, la coexistencia de varias comunidades epistémicas puede implicar un riesgo de ineficacia en la medida en que estas serian el reflejo de una falta de coordinación entre los actores. En este contexto, contrariamente a lo que sugiere Kogut (2000), las dinámicas de autorregulación, que permitirían imponer una norma, no parecen haber desempeñado su papel. Por lo tanto, es legítimo preguntarse los tipos de mecanismos institucionales que permiten la generación de un consenso sobre los conocimientos pertinentes y las normas de intercambio legítimas en este medio. Siguiendo el desarrollo de los estudios a nivel organizacional, nuestros análisis y exploraciones actuales se focalizan en el efecto de los nichos sociales, las relaciones multiplex, los desempeños económicos y la proximidad geográfica sobre la coordinación de los actores en la transferencia de conocimientos y en el proceso de aprendizaje colectivo. 


\section{Bibliografía}

Aharonson, B., Baum, J., Plunket, A. (2008) 'Inventive and uninventive clusters: The case of Canadian biotechnology', Research Policy, Vol. 37, pp.1108-1131.

Arora, A., and Gambardella, A. (1990) 'Complementarity and external linkages: the strategies of the large firms in biotechnology', The journal of industrial economics, Vol. 38, pp.361- 379.

Audretsch, D. and Stephan, P. (1996) 'Company-Scientist Locational Links: The Case of Biotechnology', The American Economic Review, pp.641-652.

Autant-Bernard, C., Mangematin, V. and Massard, N. (2006) 'Creation of Biotech in France', Small Business Economics, Vol. 26, pp.173-87.

Baum, J., Calabrese, T. and Silverman, B. (2000) 'Don't go it alone: Allieance network composition and startups' performance in Canadian biotechnology', Strategic Management J ournal, Vol. 21, pp.267-94.

Blau, P.M. (1955) The dynamics of bureaucracy, Chicago: University of Chicago Press.

Blau, P. M. (1964) Exchange and power in social life, New York: John Wiley.

Boufaden, N. and Plunket, A. (2005) 'Investigating technological and geographic proximity on forms' innovation in an immature cluster: the Paris area biotech cluster', DRUID Conference, Copenhagen.

Casper, S. (2007) 'How do technology clusters emerge and become sustainable? Social network formation and inter-firm mobility within the San Diego biotechnology cluster', Research Policy, Vol. 36, pp.438-455.

Gertler, M.S. and Levitte, Y. M. (2005) 'Local Nodes in Global Networks: The Geography of Knowledge Flows in Biotechnology Innovation', Industry and Innovation, Vol. 12, pp.487-507.

Gibbons, D. (2004) 'Friendship and Advice Networks in the Context of Changing Professional Values', Administrative Science Quarterly, Vol. 49, pp.238-262.

Gilding, M. (2008) “The tyranny of distance': Biotechnology networks and Clusters in the antipodes', Research Policy, Vol. 37, pp.1132-1144. 
Goffman, E. (1974) Frame analysis: An essay on the organization of experience, London: Harper and Row.

Kogut, B. (2000) 'The Network as Knowledge: Generative Rules and the Emergence of Structure', Strategic Management J ournal, Vol. 21, pp.405-425.

Lazega, E. (1992) Micropolitics ok Knowledge: Communication and indirect control in workgroups, Aldine-de-Gruyter, New York.

Lazega, E. (1999), ‘Le Phénomène collégial : Une théorie structurale de l'action collective entre pairs', Revue Française de Sociologie, Vol. XL, No 4, pp.639-70.

Lazega, E. (2001) The Collegial Phenomenon: The Social Mechanisms of Cooperation Among Peers in a Corporate Law Partnership, Oxford University Press, Oxford.

Lazega, E. (2004) 'Collégialité, relations d'autorité et production de biens d'apprentissage' in Delamotte, E. (ed), Du partage au marché : regards croisé sur la circulation des savoirs, Lille, pp.39-55.

Lazega, E. (2007) Réseaux sociaux et structures relationnelles, Collection QSJ?, Presses Universitaires de France.

Lazega, E. (à paraître 2008) 'Théorie de la coopération entre concurrents : Organisation, marché et analyse de réseaux', in Steiner, P. and Vatin, F. (eds), Sociologie et économie, Paris, PUF, collection Quadrige.

Lazega, E. and Van Duijn, M. (1997) 'Position in formal structure, personal characteristics and choices of advisors in a law firm: A logistic regression model for dyadic network data', Social Networks, Vol. 19, pp.375-397.

Lazega, E. and Mounier, L. (2002) 'Interdependent entrepreneurs and the social discipline of their cooperation: The research program of structural economic sociology for a society of organizations', in FAVEREAU, O. and LAZEGA, E. (eds), Conventions and Structures in Economic Organization, Edward Elgar Publishing, pp.147-199.

Lazega, E., Mounier, L., Jourda, M.-T. and Stofer, R. (2006a) 'Organizational vs. personal social capital in scientists' performance: A multi-level network study of elite French cancer researchers (1996-1998)', Scientometrics, Vol. 67, No. 1, pp. 27-44. 
Lazega, L., Lemercier, C. and Mounier, L. (2006b) 'A Spinning top model of formal organization and informal behavior: dynamics of advice networks among judges in a commercial court', European Management Review, Vol. 0, pp.1-10.

Lazega, E., Mounier, L., Snijders, T. and Tubaro, P. (2008) 'Réseaux et controverses: de l'effet des normes sur la dynamique des structures', Revue française de sociologie, Vol. 49, pp.467-498.

Lemarié, S., Mangematin, V. and Torre, A. (2001) 'Is the creation and development of biotech localized? Conclusion drawn from the French case', Small Business Economics, Vol. 17, pp.61-76.

Mangematin, V., Lemarie, S., Boissin, J.P., Catherine, D., Corolleur F., Corolini, R. and Trommetter, M. (2003) 'Development of SMEs and heterogeneity of trajectories: the case of biotechnology in France', Research Policy, Vol. 32, pp.621638.

Mcdonald, M. and Westphal, J. (2003) 'Getting by with the Advice of Their Friends: CEOs' Advice Networks and Firms' Strategic Responses to Poor Performance', Administrative Science Quarterly, Vol. 48, pp. 1-32.

Niosi, J. (2003) 'Alliances are not enough explaining rapid growth in biotechnology firms', Research Policy, Vol. 32, pp.737-750.

Pina-Stranger, Álvaro (2009) "Transfert technologique et processus collectif d'apprentissage dans l'industrie des biotechnologies en France." Sociologie Santé 30:27-49.

Pina-Stranger, Álvaro (2010) «La structure sociale de l'industrie des Biotechnologies en France: une étude des relations inter-organisationnelles au niveau inter-individuel», REDES: Revista Hispana para el Análisis de Redes Sociales. Vol.18, Numéro spécial « Réseaux sociaux et santé ».

Pisano, G. (1991) 'The governance of innovation: vertical integration and collaborative arrangements in the biotechnology industry', Research Policy, Vol. 20, pp. 237-249.

Powell, W.W., Koput, K. and Smith-Doerr, L. (1996) 'Inter-Organizational Collaboration and the locus of innovation: networks of learning in the biotechnology', Administrative Science Quarterly, Vol. 41, pp.116-45. 
Powell, W.W., Koput, K., Smith-Doerr, L. and Owen-Smith, J. (1999) 'Network position and firm performance: Organizational returns to collaboration in the biotechnology industry', Research in the Sociology of Organizations, Vol. 16, pp.129- 159.

Powell, W.W., Koput, K., Bowie, J. and Smith-Doerr, L. (2002) 'The Spatial Clustering of Science and Capital: accounting for Biotech Firm-Venture Capital relationships', Regional Studies, Vol. 36, pp.291-305.

Reynaud, J.-D. (1989) Les règles du jeu, Paris, Armand Colin.

Robins, G., Snijders, T., Wang, P., Handcock, M., Pattison, P. (2007) 'Recent developments in exponential random graph $\left(p^{*}\right)$ models for social networks', Social Networks, Vol. 29, pp.192-215.

Robins, G. and Pattison, P. (2005) 'Interdependencies and Social Processes: Generalized Dependence Structures.' in Models and Methods in Social Network Analysis, edited by Peter Carrington, P. Scott, J. and Wasserman, S., Cambridge, England: Cambridge University Press, pp.192-214

Salman, N. and Saives, A.-L. (2005) 'Indirect networks: an intangible resource for biotechnology innovation', R\&D Management, Vol. 35, pp.203-215.

Senker, J. and Sharp, M. (1997) 'Organizational learning in cooperative alliances: Some case studies in Biotechnology', Technology Analysis \& Strategic Management, Vol. 9, pp.35-51.

Snijders, T., Pattison, P., Robins, G.L. and Handcock, M., (2006) 'Newspecifications for exponential random graph models', Sociological Methodology, Vol. XX, pp. XX$\mathrm{XX}$.

Snijders, T., Steglich, C., Schweinberger, M. and Huisman, M. (2007) 'Manual for Siena version 3.11', Groningen, University of Groningen, ICS, Oxford, University of Oxford, Department of Statistics [http://stat.gamma.rug.nl/stocnet].

Sparrowe, R., Liden, R., Wayne, S. and Kraimer, M. (2001) 'Social networks and the performance of individuals and groups', Academy of Management J ournal, Vol. 44, No 2, pp. 316- 325. 
Stuart, T. and Sorenson, O. (2003) 'The geography of opportunity: spatial heterogeneity in founding rates and the performance of biotechnology firms', Research Policy, Vol. 32, pp.229-253.

Stuart, T., Ozdemir, S. and Ding, W. (2007) 'Vertical alliance networks: The case of university-biotechnology-pharmaceutical alliance chains', Research Policy, Vol. 36, pp. 477-498.

Van Duijn, M., Snijders, T. and Zijlstra, B. (2004) 'p2: a random effects model with covariates for directed graphs', Statistica Neerlandica, Vol. 58, pp.234-254.

Zijlstra, B. and Van Duijn, M. (2008) 'Manual p2, version 4.0.0.0', edition 6, August 21, Groningen, University of Groningen [http://stat.gamma.rug.nl/stocnet]. 